\title{
The Application of Chaos in Digital Water-marking and Information Hiding
}

\author{
Jing Chang ${ }^{a}$, Dong Liu ${ }^{b}$ \\ The Department of Information Science \& Technology Guangdong University of Foreign Studies \\ South China Business College, Guangdong, Guangzhou 510545, China \\ ahapipingye@163.com, ${ }^{\text {b7 } 78972493 @ q q . c o m ~}$
}

Keywords: chaos, water-marking, information hiding.

\begin{abstract}
This paper gives a brief introduction of chaos, digital water-marking and the application of chaos digital water-marking and information hiding. Then it analyzes the main difficulty in corresponding research that hidden information should have strong robustness and invisibility which contradict each other. Therefore, information hiding must, achieve solid robustness under the premise of invisibility. Such requirement is even stricter, when it comes to digital water-marking. To meet the requirement, chaotic sequence generated by chaotic mapping can be used in the scrambling treatment of hidden image, so as to achieve the purpose of hiding.
\end{abstract}

\section{Introduction}

With the rapid development of network communication technology, we are facing new opportunities and challenges in the field of convenient, fast and inexpensive digital transmission. The Internet is an open system for the general public wherein information confidentiality and system safety is not fully explored. Therefore, the protection of information content is becoming increasingly important. By virtue of the mathematical properties of the chaotic system with intelligent algorithms and the decent statistical properties of the chaotic sequences generated by the chaotic system, it is possible to provide effective solutions [1] for information management and protection based on the chaos theory in combination with digital water-marking and digital hiding techniques.

Compared with the typical text, digital images can carry more information and hide other covert digital information within them by virtue of their inherent data features. With close relation to information hiding, digital water-marking becomes a new type of digital copyright protection technology which uses data hiding techniques to hide specific information in digital products. It can be used to identify and protect the right of authorship and copyright in digital products, so as to prove that the products are authentic and reliable.

\section{The Application of Chaos in Digital Water-marking and Information Hiding}

\subsection{Features of Chaos Theory}

Chaos is a special form of motion contained in Nonlinear Dynamic Systems and a pseudo-random phenomenon between deterministic and stochastic phenomena. Its main features include high sensitivity to initial value and noise, pseudo randomness, unpredictability, etc. In respect of application to the field of digital information hiding and digital water-marking techniques, chaos is mostly used in chaotic systems which are applied for pretreatment of digital and water-marking information to be hidden, after which it will be possible to embed the digital or water-marking signals through the use of certain techniques. By virtue of the sensitivity of chaos to initial values, it is possible to conduct scrambling treatment on the embedded signals, which is the pretreatment method for hiding the digital and water-marking information.

Due to the singularity and complexity of chaotic systems, there is no unified definition of chaos as of now and the existing definitions only reflect the characteristics of chaotic motion from different aspects. Li-Yorke's Chaos is a mathematical definition of chaos with greater impact and it is from the aspect of interval mapping; Melnikov's definition of chaos is mainly based on the pioneering research 
of Smale's horseshoe theory; while Devaney's is a chaos definition given from the aspect of topology [2].

\subsection{Information Hiding}

Information hiding is a method by which certain secret information is secretly hidden in another publicly-available information content and then transmitted through the transmission of shared information. Information security technique based on information hiding can hide not only the content of the information but also its existence. The security function of such technique comes from its perception paralysis effect on a third-party, which makes it difficult for a potential detector or illegal interceptor to determine the existence of confidential information from public information and to intercept such confidential information, and the confidential information is protected as a result. Extensive application of multimedia technology extends the development field for information hiding. Fig. 1 below is a general model of information hiding [3].

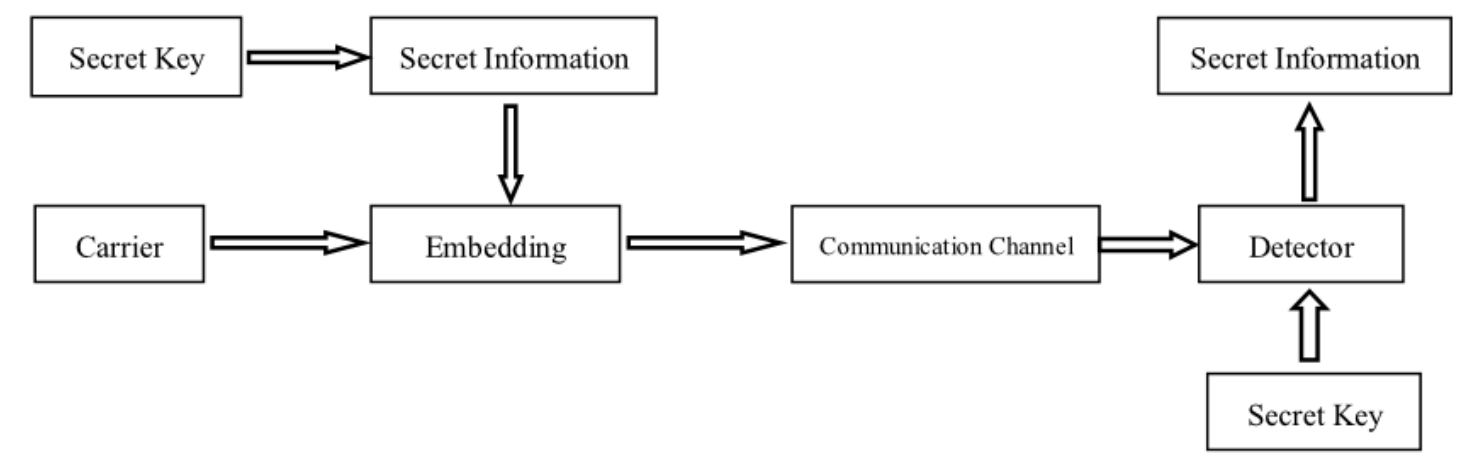

Fig. 1 Information Hiding Model

Within the model, secret information is the information to be hidden and public information has become the carrier information. The information hiding process is generally controlled by a secret key; the embedding algorithm is used to hide secret information in public information, which is transmitted through the channels as the hidden carrier. Then the detector will use the secret key to recover the secret information from the hidden carrier.Considering its purposes, it is required for information hiding technique to bear five characteristics, i.e., robustness, non-detectability, transparency, security and self-recovery [4].

\subsection{Digital Water-marking}

Cox et al. [5]defined water-marking as "an operation behavior to embed information in the works in an imperceptible manner". Digital water-marking is a distinguishable digital signal or pattern that is permanently embedded in other data without affecting the availability of the host data [6]. Although requirement for digital water-marking varies depending on its specific application, such techniques generally shall have the following characteristics [7]:

(1) Provability

Watermarks can serve as a complete and reliable evidence for the ownership of copyrighted information products. Water-marking algorithm can be used to get the owner's relevant information embedded in the object protected, and extract such information when required.

(2) Imperceptibility

Embedded watermark causing conversion of carrier data should be unnoticeable to the viewer's visual or auditory system. Ideally, watermark and the original carrier should be visually identical, which should be a requirement to be met by most water-marking algorithms.

(3) Robustness

Watermarks should be able to withstand a mass of physical and geometric distortion. Even after withstanding such operations, robust water-marking algorithm should still be capable of extracting embedded watermarks from the carriers or proving the existence of such watermarks. Robustness of digital water-marking algorithm reflects the algorithm's capability to withstand a variety of attacks. Robustness is directly dependent on the embedding strength, which is related to image degradation [8]. A good digital water-marking system should be able to make the watermarked original image 
relatively strong in robustness with minimal visual distortion. The purpose of the attack is to change the data so as to make the embedded watermarks unidentifiable, and watermarks made with effective algorithms must be difficult to get rid of. However, if the information can only be partly retrieved, an operation attempting to undermine the watermarks will result in serious degradation of the image quality.

\subsection{Image Scrambling Techniques for Chaotic Mapping}

Chaotic sequence can be used to scramble the hidden image, i.e. the generated chaotic sequence can be used to scramble the position of each pixel in the hidden images and make such pixels rearrange. Image scrambling for 2-dimensional chaotic mapping can be viewed as space conversion of points on a planar area [9]. Arnold conversion is a mocking-up type of non-linear conversion with periodicity and can be viewed as an endomorphism in the study of torus.

$$
\left(\begin{array}{l}
x_{n+1} \\
y_{n+1}
\end{array}\right)=\left(\begin{array}{ll}
a_{11} & a_{12} \\
a_{21} & a_{22}
\end{array}\right)\left(\begin{array}{l}
x \\
y
\end{array}\right)(\bmod 1)
$$

In Formula 1, $\left(\begin{array}{ll}a_{11} & a_{12} \\ a_{21} & a_{22}\end{array}\right)$ is the conversion matrix, which is denoted as A; mod indicates mocking-up operation and the modulo operation regulation for the matrix should be applicable to element mocking-up; $\left(\begin{array}{l}x \\ y\end{array}\right)$ and $\left(\begin{array}{l}x_{n+1} \\ y_{n+1}\end{array}\right)$ indicates points on torus. Digital images can be considered as the sample value of a function at a point in a discrete grid, through which an evidencing image can be obtained. Value of the element in the matrix shall be conforming to the gray scale value or GRB color component value of the same point. Where $N \times N$ is the image size, a scrambled image can be obtained after several iterations.

$$
\left(\begin{array}{l}
x_{n+1} \\
y_{n+1}
\end{array}\right)=\left(\begin{array}{cc}
1 & 1 \\
k & k+1
\end{array}\right)\left(\begin{array}{l}
x \\
y
\end{array}\right)(\bmod N), k \in[1, N]
$$

In Formula 2, $x, y \in\{0,1,2, \ldots, N-1\}, N$ is the order of digital image matrix and $(\bmod N)$ indicates mock-up operation. Since the cycle of 2-dimensional chaotic conversion will vary due to difference in order of matrix $N$ and the value $k$ in Formula 2, and it is best to shorten the conversion cycle when there is need to minimize the cost arising out of 2-dimensional chaotic conversion. Arnold conversion cycle is associated with the value $k$ in Formula 2 as well; change in value $k$ is in fact the change in the slope of each track during the conversion, which in turn changes the cycle. Therefore, minimal value $k$ can be used for the scrambling conversion cycle.

Order of matrix $N$ is not proportional to 2-D chaotic conversion cycle, wherefore the order $N$ with relatively shorter Arnold conversion cycle shall be selected when determining on the size of the digital water-marking image. If the image size is determined, value $k$ can be appropriately selected to reduce the cycle of scrambling change. In practice, it is required to change the value $k$ as needed and use it as the secret key for watermark scrambling. Besides, different $k$ values can be obtained by calculating the optimal number of iterations while the secret key is being used, so as to make the best scrambling of image; scrambled image will enhance the effectiveness of the watermark in resisting cropping attack in visual aspects, for the scrambling of image dispersed the relatively concentrated original pixels and the extracted watermark image will have better visual effect.

\section{Conclusion}

In respect of application to the field of digital information hiding and digital water-marking techniques, chaos is mostly used in chaotic systems which are applied for pretreatment of the digital and water-marking information to be hidden. After that, certain techniques can be used to embed the digital or water-marking signals. Providing the overview of the concepts and principles involved in the aforesaid process and making corresponding theoretical analysis will facilitate the understanding of the application of chaos to digital information hiding and water-marking techniques. 


\section{References}

[1] Chen Yonghong, Huang Xiyue. Public Digital Water-marking Techniques Based on Chaotic Mapping and Matrix Singular Value Decomposition. Computer Simulation, 1.2005: 138-141.

[2] Guan Xinping Et al. Chaos Control and its Application in Secure Communication. National Defense Industry Press, Beijing, 2002

[3] Fridrich J. Applications of data hiding in digital images. In: the ISPAC'98 Conference in Melbourne,Australia.1998,11

[4] Yi Kaixiang, Shi Jiaoying, Sun Xin. Advances in the Study of Digital Water-marking Technology. China Image and Graphics, 2.2001: 111-117

[5] Cox I J, Miller M L, Bloom J A. Digital Water-marking. Translated by Wang Ying, Huang Zhibei. Electronic Industry Press, Beijing, 2003

[6] Chen Mingqi, Niu Xinxin, Yang Yixian, Development and Application of Digital Water-marking. Journal of Communications. 2001, 22 (5): 71-79

[7] Mu Xiaomin, Zhang Dehui, Zhu Chun et al. Analysis on the Robustness of Digital Water-marking against Stirmark Attack. Telecommunication Engineering, 3.2004: 52-56

[8] Xia Wei, Lu Hongwei, Zhao Xiaoxia. Robust Digital Image Water-marking Technology. Small Micro-computer System. 2.2011: 356-360

[9] Xia Wei, Lu Hongwei, Xie Changsheng,et al.Improvement-based Robust Digital Water-marking Method with Invariant Centroid and Wavelet Domain. Computer Science, 4.2011: 278-281 